\title{
Special Meeting Sections for the 6th ASM Conference on Beneficial Microbes
}

\author{
George A. O'Toole
}

Geisel School of Medicine at Dartmouth, Hanover, New Hampshire, USA

$\mathrm{n}$ this issue of the Journal of Bacteriology, we present sections dedicated to the 6 th ASM Conference on Beneficial Microbes, held in Seattle, WA, from 9 to 12 September 2016. These special sections contain five minireviews and one original article and are highlighted by a terrific cover image.

In some ways, the preponderance of minireviews reflects the reality of this growing field - one that focuses on "the good microbes." That is, this is a field that has recently been rediscovered. The early history of microbiology did include significant efforts directed toward understanding beneficial microbes, particularly those organisms with a role in the food industry. Indeed, many early departments had names with some variation of "Food Microbiology." As microbial research has evolved, and particularly over the past few decades, it has been driven largely by a quest to understand pathogenic microbes and their impact on the host. In many ways, the resurgence of studies delving into the biology of beneficial microbes has allowed the discipline to rediscover its roots.

As you will see, the theme of many of the articles in these special sections can be summed up as "polymicrobial interactions and models to study these polymicrobial communities." As we learn more about how the microbiota impacts our underlying health, it is not surprising that more and more researchers have tried to develop systems that leverage knowledge gained from pure culture work and apply this information to understanding mechanisms of microbe-microbe interactions. I also argue that by simply putting microbes together in the same time and place one can uncover new microbial biology that has yet to be revealed in a pure culture systemthis idea is also covered in some of these articles.

Some interesting new biology is highlighted in these special sections, and I anticipate that future Benefical Microbes conferences will continue to make important contributions to our understanding of how microbes play a positive role in our lives and in the environment.

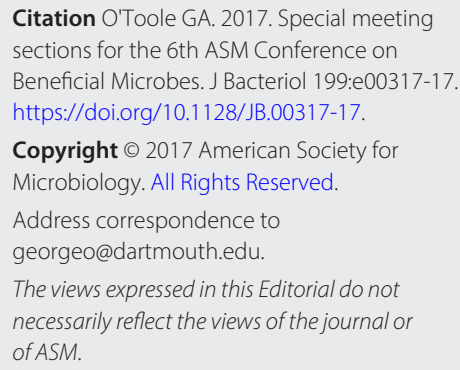

